Supporting Information

\title{
Transition State Compressibility and Activation Volume of Transient Protein Conformational Fluctuations
}

Matthias Dreydoppel ${ }^{1}$, Britta Dorn ${ }^{1}$, Kristofer Modig 2 , Mikael Akke ${ }^{2}$ and Ulrich Weininger ${ }^{1, *}$

${ }^{1}$ Institute of Physics, Biophysics, Martin-Luther-University Halle-Wittenberg, D-06120 Halle (Saale), Germany

${ }^{2}$ Division of Biophysical Chemistry, Center for Molecular Protein Science, Department of Chemistry, Lund University, P.O. Box 124, SE-22100 Lund, Sweden

* Ulrich Weininger

Email: ulrich.weininger@physik.uni-halle.de

Phone: +493455528555 


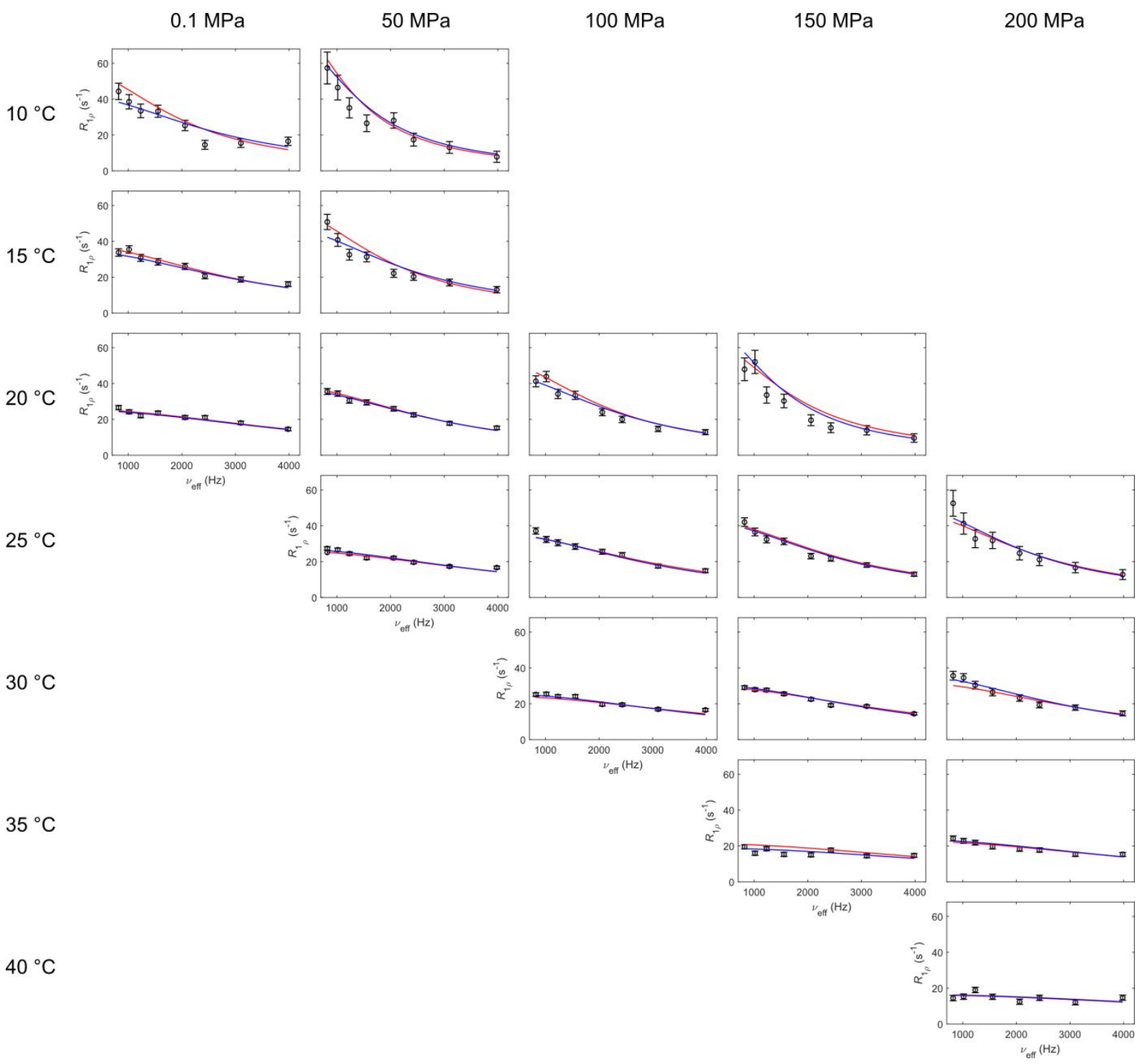

Figure S1: ${ }^{13} \mathrm{C}$ aromatic $R_{1 \rho}$ relaxation dispersion profiles for $\mathrm{F} 52 \varepsilon$ recorded on-resonant $\left(\theta>85^{\circ}\right)$ on a $5 \mathrm{mM}$ sample of GB1 at $\mathrm{pH} 7.0$ and a static magnetic field strength of $14.1 \mathrm{~T}$. The relaxation dispersions were fitted using a fixed population $p_{1}=p_{2}=0.5$ and $\Delta \delta$ fixed at the value measured from HSQC spectra under slow-exchange conditions. Blue lines depict global fits of all temperatures at each pressure, fitted with the restrictions: $k_{\text {flip }}\left(T_{\text {high }}\right)>k_{\text {flip }}$ $\left(T_{\text {low }}\right)$, and $R_{2,0}\left(T_{\text {high }}\right) \leq R_{2,0}\left(T_{\text {low }}\right)$. Derived ring flip rate constants $\left(k_{\text {flip }}\right)$ are given in Table 1 . Red lines depict the global fit of all relaxation dispersions directly to the model given by Eq. (6), with the restriction: $R_{2,0}\left(T_{\text {high }}\right) \leq R_{2,0}\left(T_{\text {low }}\right)$ for sets of equal pressure. 

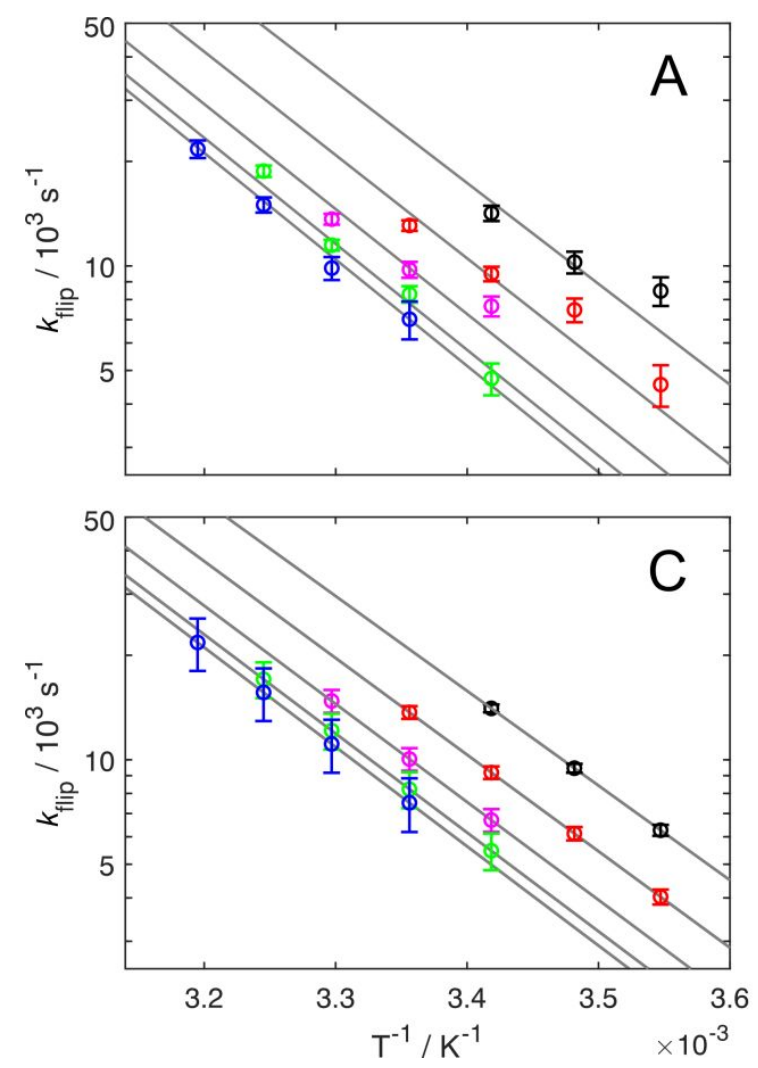

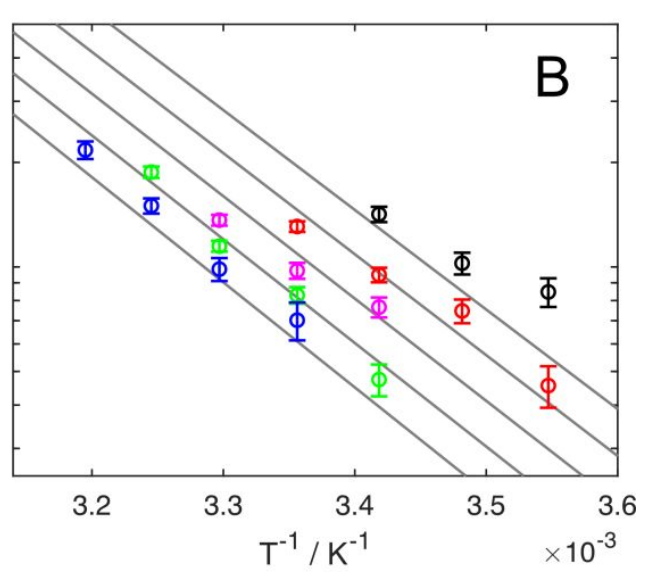

Figure S2: Temperature and pressure dependence of flip rates. $k_{\text {flip }}$ is plotted as a function of $1 / T$ for F52 at pressures of $0.1 \mathrm{MPa}$ (black), $50 \mathrm{MPa}$ (red), $100 \mathrm{MPa}$ (magenta), $150 \mathrm{MPa}$ (green) and $200 \mathrm{MPa}$ (blue). The solid lines represent the fitted model including the activation parameters $\Delta^{\ddagger} G_{0}, \Delta^{\ddagger} S_{0}$, and $\Delta^{\ddagger} V_{0}$ at the reference point of $0.1 \mathrm{MPa}$ and $293 \mathrm{~K}$, as well as $\Delta^{\ddagger} \kappa^{\prime}$, according to Eq. (6). (A) Flip rates derived from $R_{1 \rho}$ relaxation dispersions, based on simultaneous fits of all data (all temperatures) at a given pressure using the restrictions $k_{\text {flip }}$ $\left(T_{\text {high }}\right)>k_{\text {flip }}\left(T_{\text {low }}\right)$, and $R_{2,0}\left(T_{\text {high }}\right) \leq R_{2,0}\left(T_{\text {low }}\right)$. (B) Flip rates as in A, fitted with the restriction $\Delta^{\ddagger} \boldsymbol{\kappa}^{\prime}=0$. (C) Global fitting of $\Delta^{\ddagger} G_{0}, \Delta^{\ddagger} S_{0}, \Delta^{\ddagger} V_{0}$, and $\Delta^{\ddagger} \boldsymbol{K}^{\prime}$ directly to all temperature and pressure dependent relaxation dispersion data. In $\mathrm{C}$, flip rates are subsequently calculated from the fitted thermodynamic parameters, and error bars are derived by error propagation; for this reason, error bars are larger for data points further from the reference point. 


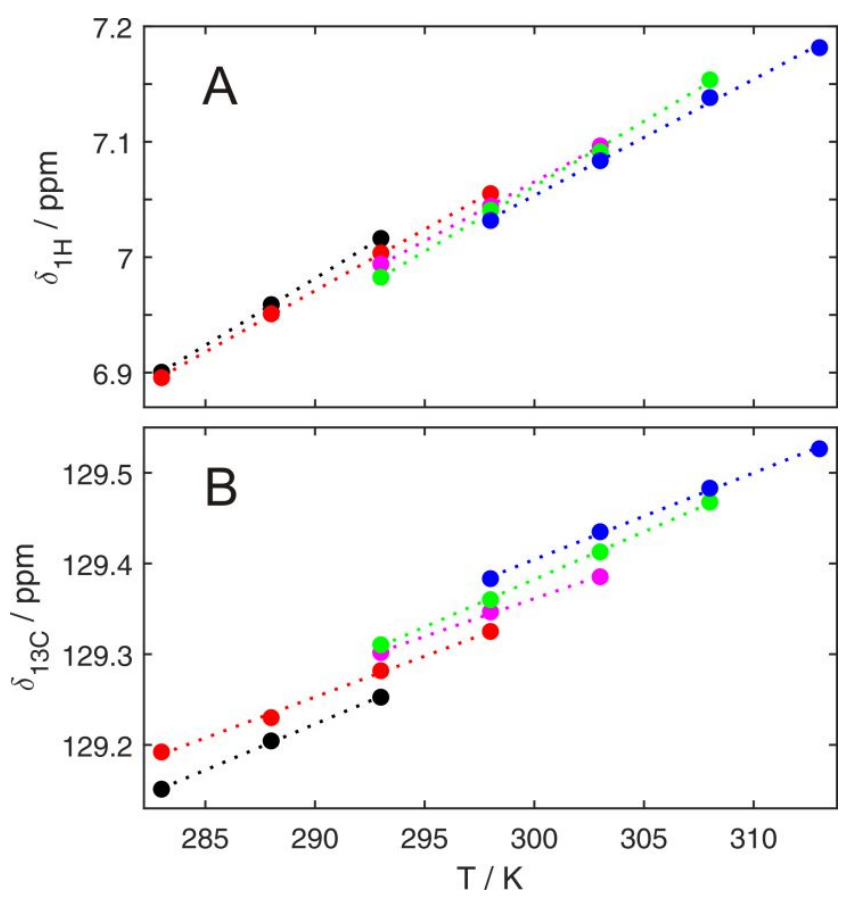

Figure S3: Temperature and pressure dependence of chemical shifts of the averaged signal of F52 $\varepsilon . \delta_{1 \mathrm{H}}(\mathrm{A})$ and $\delta_{13 \mathrm{C}}(\mathrm{B})$ are plotted as a function of $T$ at pressures of $0.1 \mathrm{MPa}$ (black), $50 \mathrm{MPa}$ (red), $100 \mathrm{MPa}$ (magenta), $150 \mathrm{MPa}$ (green) and $200 \mathrm{MPa}$ (blue). Dotted lines depict linear fits of $\delta(T)$ at the respective pressures. Slopes of the linear fits range from 0.010 to $0.011 \mathrm{ppm} / \mathrm{K}$ for ${ }^{1} \mathrm{H}$ and from 0.008 to $0.010 \mathrm{ppm} / \mathrm{K}$ for ${ }^{13} \mathrm{C}$. At temperatures of 293 and $298 \mathrm{~K}$, shift changes with pressure are less than or equal to $0.22 \mathrm{ppm} / \mathrm{GPa}$ for ${ }^{1} \mathrm{H}$ and $0.39 \mathrm{ppm} / \mathrm{GPa}$ for ${ }^{13} \mathrm{C}$, respectively. 